\title{
INVENTÁRIO DO SEXISMO AMBIVALENTE EM BRASILEIROS: SUA ACURÁCIA ESTRUTURAL.
}

INVENTORY OF AMBIVALENT SEXISM IN BRAZILIAN: ITS STRUCTURAL ACCURACY.

DOI: 10.22199/S07187475.2011.0002.00005

Recibido: 15 de Julio del 2011 | Aceptado: 29 de Agosto del 2011

NILTON S. FORMIGA

(UNIVERSIDADE FEDERAL DA PARAíBA, Faculdade Maurício de Nassua - JP, Brasil).

\begin{abstract}
RESUMO
0 sexismo ambivalente é compreendido como um conjunto de estereótipos sobre a avaliação cognitiva, afetiva e atitudinal acerca do papel apropriado na sociedade dirigida aos indivíduos de acordo com o sexo, avaliado no caso das mulheres como sexismo hostil e sexismo benévolo. Neste estudo, tem-se como objetivo comprovar a manutenção da estrutura bidimensional desse constructo. Quatro amostras de homens e mulheres, com idade de 16 e 60 anos, responderam 0 inventário de sexismo ambivalente; este, constituído por 22 itens e respondidos em escala de cinco pontos, tipo Likert. 0 inventário foi aplicado individualmente nas instituições de ensino fundamental, médio e universitário, garantindo o anonimato e a confidencialidade das respostas dos sujeitos. Os indicadores psicométricos encontrados, calculados no programa AMOS GRAFICS 7.0, confirmaram a existência dos dois fatores, em todas as amostras se assemelhando aos encontrados nos estudos brasileiros e transculturais.
\end{abstract}

PALABRAS CLAVE: Sexismo; Análise estrutural; Acurácia.

\section{ABSTRACT}

Ambivalent sexism refers to a series of stereotypes that affect the cognitive, affective and attitudinal assessment of individuals in relation to their gender roles. In the case of women, ambivalent sexism comprises two dimensions, hostile sexism and benevolent sexism. The objective of this study was to verify the bi-dimensional structure of the construct. Four samples of men and women, between the ages of 16 and 60 , completed the ambivalent sexism inventory. This instrument consists of 22 items and the answers are given using a five-point Likert scale. The inventory was administered individually in elementary, high school and university institutions. Anonymity and confidentiality of the information provided was guaranteed to all subjects. The psychometric indicators -calculated with the program AMOS GRAFICS 7.0- confirmed the existence of two factors in all samples, supporting findings reported by previous Brazilian and other transcultural studies.

KEY WORDS: Sexism; Structural analysis; Assessment.

1. 0 autor é doutor em Psicologia Social pela Universidade Federal da Paraíba. Atualmente, é professor no curso de Psicologia na Faculdade Mauricio de Nassau - JP. Endereço para correspondência: Rua Juiz Ovídio Gouveia, 349. Pedro Gondim. CEP.: 58031 -030. João Pessoa - PB. E-mail: nsformiga@yahoo.com 
INTRODUÇÃO

O tema sobre a discriminação em relação à mulher permite inúmeras especulações em vários espaços das Ciências Humanas e Sociais (Aguiar, 1997). As causas e conseqüências que podem ser hipotetizadas e defendidas, porém, somente poderá ser conhecida sua verdadeira extensão ao considerar, diretamente, os agentes que fomentam o fenômeno do preconceito, isto é, as atitudes de homens e mulheres da sociedade civil.

O problema do preconceito ainda se repercute na dinâmica social e científica; isto se deve, por se debater que tal construto tem desaparecido na interpessoalidade da sociedade devido ao avanço tecnologico, profissional, educacional, político e social atualmente, bem como, pela possibilidade de controle sobre qualquer manifestação discriminatória, principalmente, em relação à mulher. De fato, esse fenômeno nunca sumiu, apenas adquiriu-se novas formas de manifestação, as quais são sutis, disfarçadas, camufladas (Pettigrew \& Meertens, 1995; Swim, Aikin, Hall \& Hunter, 1995; Tougas, Brown, Beaton \& Joly, 1995). Metaforicamente, 0 preconceito pode ser comparado a um iceberg, cuja maior parte fica encoberta e apenas pode ser ver sua ponta, enquanto o que está submerso além de ser enorme, requer instrumentos e mecanismos especializados para que se possa ter em conta o tamanho e o possível risco para a natureza (Munanga, 2002).

Essas novas formas do preconceito podem ser analogamente consideradas a parte submersa, aquela que não se vê ou "finge" não vê. Algo semelhante pode ser comparado ao preconceito frente às mulheres, já que elas são observadas como grupo "minoritário" (Formiga \& cols., 2004), pois, salienta-se uma percepção na dinâmica psicossocial que 0 preconceito frente a elas "não ocorre no Brasil" devido à expressiva cordialidade e aos atributos de feminilidade tão salientes na dinâmica social que se têm elas no Brasil. Esses atributos estereotipam as mulheres como uma categoria 'satisfeita' com o espaço social ocupado e que elas são sensíveis, maternas, emotivas, etc. (Formiga \& Camino, 2001).

Segundo Formiga, Gouveia e Santos (2002), as opiniões, atitudes e pensamentos sobre o papel da mulher em diferentes facetas da sociedade são cruciais no momento de compreender formas e conteúdos de um problema ainda muito antigo sobre 0 preconceito. Faz-se necessário, a contínua exploração de um instrumento que avalia o preconceito em relação às mulheres, este é denominado de sexismo. De acordo com Glick e Fiske (1996), esse fenômeno discriminatório tem se apresentado sob novas formas modernas, as quais esses autores consideram-na em sua dinâmica social uma forma ambivalente. Concretamente, 0 sexismo ambivalente é compreendido como um conjunto de estereótipos sobre a avaliação cognitiva, afetiva e atitudinal acerca do papel apropriado na sociedade dirigida aos indivíduos de acordo com 0 sexo. As formas de sexismo são ambivalentes, não somente porque são indiretas, mas também, por acarretar emoções negativas e positivas, as quais de dupla valência afetivas, principalmente, quando se considera sua prática e expressão discriminatória tradicional, as quais se apresentam como:

- Sexismo Hostil é uma expressão flagrante, aberta e explícita do preconceito em relação às mulheres. Este tem sido extensamente tratado (Glick \& Fiske, 1996, 2001), porém não permite compreender totalmente a direção que toma 0 sexismo na sociedade atual. Esta forma de sexismo, justificado a luz da busca de igualdade em direitos e deveres entre os gêneros (Siano, 2000), evidencia crenças e práticas típicas de pessoas que 
consideram as mulheres inferiores aos homens, refletindo antipatia e intolerância em relação ao seu papel como figura de poder e decisão;

- Sexismo Benévolo se constitui a partir das concessões e tratamentos diferenciados entre homens e mulheres, referindo-se a uma atitude positiva, aparentemente não preconceituosa em relação à mulher, porém descrevendo-a como pessoa frágil, necessitando de atenção, proteção e provisão por parte dos homens (Pettigrew \& Meertens, 1995).

No Brasil, o inventário de sexismo ambivalente, desenvolvido por Glick e Fiske (1996), foi adaptado por Formiga, Gouveia e Santos (2002) em uma amostra de jovens universitários paraibanos; a partir de uma análise fatorial exploratória, os índices de consistência interna e relação itens-fator foram semelhantes aos encontrados por Glick e Fiske (1996). Ferreira (2004) desenvolveu um trabalho semelhante, verificando, a partir de uma análise exploratória, a bi-dimensionalidade do construto do sexismo ambivalente, obtendo consistência interna aceitável. Na mesma direção, Formiga (2005) comprovou mais uma vez que, tanto a relação itens-fator quanto os índices de consistência interna, os resultados foram semelhantes aos observados nas pesquisas já desenvolvidas com esse instrumento.

Mas, segundo Formiga (2011), essa análise exploratória do inventário nos estudos supracitados, pauta-se estritamente nos dados obtidos desconsiderando um modelo teórico fixo que oriente a extração das dimensões latentes e esta, muito menos têm 0 poder de apresentar qualquer indicação sobre a bondade de ajuste do modelo. Assim, a partir de uma análise fatorial confirmatória e a modelagem de equação estrutural no programa AMOS 7.0, comprovou-se, empírica e teoricamente, que o inventário de sexismo ambivalente, se estruturou nas mesmas dimensões encontradas anteriormente, confirmando os estudos exploratórios.

Neste estudo, pretende-se avaliar a partir da modelagem de equação estrutural 0 inventário de sexismo ambivalente em relação à manutenção da bidimensionalidade desse construto. Assim, espera-se que, em diferentes contextos sócio-econômicos considerando 0 pressuposto teórico e metodológico do inventário do sexismo, este seja comprovado, tanto buscando garantir sua robustez psicométrica quanto consistência estrutural.

\section{MÉTODO}

\section{Amostra}

Quatro amostras com sujeitos com idades de 13 e 60 anos, do sexo masculino e do sexo feminino, das cidades de João Pessoa - PB e Palmas - TO compuseram o estudo. Todas elas foram distribuídas igualmente nos níveis fundamental e médio da rede privada e pública de educação de cada cidade. $\mathrm{Na}$ primeira amostra $\left(\mathbf{N}_{1}\right) 287$ sujeitos da cidade de João Pessoa - PB, com média de idade de 27,29 anos; a segunda amostra $\left(\mathbf{N}_{\mathbf{2}}\right)$ foi composta de 203 sujeitos da cidade de Palmas - TO, com faixa etária de 23 anos; na terceira amostra $\left(\mathbf{N}_{3}\right)$ com 217 sujeitos da cidade de João Pessoa - PB, apresentando uma média etária de 24,50 anos; por fim, a quarta amostra $\left(\mathbf{N}_{4}\right)$ com 208 sujeitos da cidade de João Pessoa - PB, com faixa etária de 22 anos.

Tal amostra foi não probabilística, e sim intencional; pois além do propósito de garantir a validade interna dos instrumentos da pesquisa, era assegurada a possibilidade de realizar as análises estatísticas que permitissem estabelecer os critérios preditivos entre as variáveis estudadas. 
Instrumento

Os participantes responderam um questionário constando de duas partes:

- Inventário de Sexismo Ambivalente, ISA. Elaborado originalmente em língua inglesa (Glick \& Fiske, 1996) e adaptado por Formiga, Gouveia e Santos (2002) e Formiga (2005) para o contexto brasileiro. Este instrumento é composto por 22 itens (ver anexo) que avaliam as atitudes em relação às mulheres a respeito das duas dimensões do sexismo: hostil (por exemplo, As mulheres feministas estão fazendo exigências completamente sem sentido aos homens; A maioria das mulheres não apreciam completamente tudo o que os homens fazem por elas) e benévolo (por exemplo, As mulheres devem ser queridas e protegidas pelos homens; Muitas mulheres se caracterizam por uma pureza que poucos homens possuem). Para respondê-lo a pessoa deve ler cada item e indicar 0 quanto está de acordo com o conteúdo expresso, utilizando uma escala de quatro pontos, tipo Likert, com os seguintes extremos: 1 = Discordo Totalmente e $\mathbf{5}=$ Concordo Totalmente. Em um estudo original desenvolvido por Formiga, Gouveia e Santos (2002), a partir de uma análise fatorial confirmatória, 0 inventário apresentou parâmetros psicométricos para a população brasileira com os seguintes indicadores de bondade de ajuste: $\underline{\mathrm{GFI}}=$ 0,77 e $\underline{\text { AGFI }}=0,72 ; \chi_{-}^{2} / \mathrm{gl}=3,18 ; \underline{\mathrm{RMSR}}=$ 0,10 .

- Caracterização Sócio-Demográfica.

Uma folha separada foi anexada ao instrumento prévio, onde eram solicitadas informações de caráter sócio-demográfico (idade, sexo, estado civil, etc.).

\section{Procedimento}

Procurou-se definir um mesmo procedimento padrão que consistia em aplicar o ISA individualmente, na sala de aula, a cada sujeito. Aplicadores devidamente treinados ficaram responsáveis pela coleta dos dados; após conseguir a permissão dos transeuntes quando abordados, se apresentava como interessado em conhecer as opiniões e os comportamentos das pessoas no dia a dia, solicitando a colaboração voluntária dos mesmos no sentido de responderem um questionário breve.

Foi-lhes dito que não havia respostas certas ou erradas, e que respondessem ao mais sincero possível após 0 aplicador ter finalizado sua afirmativa contida no instrumento; a todos era assegurado o anonimato das suas respostas, que seriam tratadas em seu conjunto. Desta forma, contando com as instruções necessárias para que possam ser respondidos, os pesquisadores esteve presente durante toda a aplicação para 0 esclarecimento das dúvidas que se fizesse indispensável. Um tempo médio de 30 minutos foi suficiente para concluir essa atividade.

Nos estudos anteriores, utilizou-se 0 pacote estatístico SPSSWIN, em sua versão 15.0, para tabular os dados e realizar as análises estatísticas descritivas e os cálculos referentes ao Alfa de Cronbach $(\alpha)$. Apesar da garantia na relação item-fator, na análise exploratória e as correlações internas entre fatores de cada escala encontrada nos estudos Glick e Fiske (1996), Formiga, Gouveia e Santos (2002), Ferreira (2004) e Formiga (2005) elas se baseiam apenas nos dados obtidos, desconsiderando um modelo teórico fixado, capaz de orientar a extração das dimensões latentes, justamente, porque nas análises exploratórias não é possível apresentar qualquer indicação sobre a bondade de ajuste do modelo.

Essas técnicas - a de bondade de ajuste - têm a clara vantagem de levar em conta a teoria para definir os itens pertencentes a cada fator, bem como, apresenta indicadores de bondade de ajuste que permitem decidir objetivamente sobre a 
validade de construto da medida analisada. Com isso, efetuou-se uma Análise Fatorial Confirmatória (AFC), gerando Modelo de Equação Estrutural (SEM) a partir do AMOS GRAFICS (versão 7.0) para comprovar, com maior robustez, a estrutura do inventário de sexismo ambivalente.

Para realização da análise fatorial confirmatória, pretendeu-se testar a adequação do modelo quanto a sua unidimensionalidade e bidimensionalidade. Considerou-se como entrada a matriz de covariâncias, tendo sido adotado 0 estimador ML (Maximum Likelihood). Este tipo de análise estatística é mais criterioso e rigoroso do que 0 anterior - Principais Componentes - (PC) utilizado nos estudos anteriormente citados; isto permite testar diretamente uma estrutura teórica, como a proposta pelos autores que adaptaram 0 mesmo inventário para o Brasil.

Esta análise apresenta alguns índices que permitem avaliar a qualidade de ajuste do modelo proposto (Byrne, 1989; Hair, Anderson, Tatham \& Black, 2005; Kelloway,1998; Tabachnick \& Fidell, 1996; van de Vijver \& Leung, 1997), por exemplo:

- $0 x^{2}$ (qui-quadrado) testa a probabilidade do modelo teórico se ajustar aos dados; quanto maior este valor, pior o ajustamento. Este tem sido pouco empregado na literatura, sendo mais comum considerar sua razão em relação ao grau de liberdade ( $X^{2} / g$. .I.). Neste caso, valores até 3 indicam um ajustamento adequado.

- Raiz Quadrada Média Residual $(R M R)$, que indica o ajustamento do modelo teórico aos dados, na medida em que a diferença entre os dois se aproxima de zero (Joreskög \& Sörbom, 1989).

- O Comparative Fit Index (CFI) compara, de forma geral, o modelo estimado e 0 modelo nulo, considerando valores mais próximos de um como indicadores de ajustamento satisfatório (Hair, Anderson, Tatham \& Black, 2005).

- O Goodness-of-Fit Index (GFI) e o Adjusted Goodness-of-Fit Index (AGFI) são análogos ao $\mathrm{R}^{2} \mathrm{em}$ regressão múltipla. Portanto, indicam a proporção de variânciacovariância nos dados explicada pelo modelo. Estes variam de 0 a 1 , com valores na casa dos 0,80 e 0,90 , ou superior, indicando um ajustamento satisfatório.

- A Root-Mean-Square Error of Approximation (RMSEA), com seu intervalo de confiança de $90 \%$ (IC90\%), é considerado um indicador de "maldade" de ajuste, isto é, valores altos indicam um modelo não ajustado. Assume-se como ideal que o RMSEA se situe entre 0,05 e 0,08, aceitando-se valores de até 0,10 (Garson, 2003; Kelloway, 1998).

- O Expected Cross-Validation Index (ECVI) e o Consistent Akaike Information Criterion (CAIC) são indicadores geralmente empregados para avaliar a adequação de um modelo determinado em relação a outro. Valores baixos do ECVI e CAIC expressam 0 modelo com melhor ajuste.

\section{RESULTADOS}

A partir das análises exploratórias dos estudos no Brasil (Formiga, Gouveia \& Santos, 2002; Ferreira, 2004; Formiga, 2005), bem como, da análise confirmatória (Formiga, 2010), buscou-se um maior rigor e critério psicométrico no presente estudo para a estrutura do inventário de sexismo ambivalente. Para isso efetuou-se com 0 pacote estatístico AMOS 7.0 uma análise 
fatorial confirmatória e modelagem de equação estrutural para as quatro amostras; hipotetizou-se a bifatorialidade do modelo de acordo com o que foi proposto nos estudos citados anteriormente.

A fim de testar a estrutura fatorial do Inventário de Sexismo Ambivalente considerou-se os seguintes modelos comparativos: (a) Modelo 1: unifatorial, em que todos os itens da atitude anômica apresentam saturação em um único fator $\mathrm{e}$ (b) Modelo 2: uma estrutura com dois fatores, de acordo como já foi encontrado em outros estudos descrito previamente e que te a estrutura item-fator da seguinte forma: Sexismo Benévolo - ISA 01, ISA 03, ISA 06, ISA 08, ISA 09, ISA 12, ISA 13, ISA 17, ISA 19, ISA 20, ISA 22 e o Sexismo Hostil - ISA 02, ISA 04, ISA 05, ISA 07, ISA 10, ISA 11, , ISA 14, ISA 15, ISA 16, ISA 18, ISA 21.

Optou-se por deixar livre as covariâncias (phi, $\varphi$ ) entre os fatores. Os indicadores de qualidade de ajuste de cada modelo se mostraram próximas as recomendações apresentadas na literatura (Byrne, 1989; Tabachnick \& Fidell, 1996; van de Vijver \& Leung, 1997). Os resultados obtidos nestas análises, observados, em negrito, na tabela 1 , revelam que 0 melhor modelo para 0 Inventário de Sexismo Ambivalente foi 0 modelo bifatorial, em todas as amostras. Especificamente, os seguintes indicadores da qualidade de ajuste, apresentaram a seguinte razão: amostra $1\left(X^{2} / \mathrm{gl}=1,01, \mathrm{GFI}\right.$ $=0,95, A G F I=0,92, C F I=0,99$, RMSEA $(90 \% / C)=0,01, C A I C=718,91$ e $E C V I=$ 1,18); amostra $2\left(X^{2} / \mathrm{gl}=0,89, G F I=0,94\right.$, $A G F I=0,91, C F I=1,00, R M S E A(90 \% / C)=$ $0,01, \quad C A I C=712,45$ e $E C V I=1,60)$; amostra $3\left(X^{2} / \mathrm{gl}=0,97, \mathrm{GFI}=0,96, A G F I=\right.$ $0,94, C F I=1,00, R M S E A(90 \% I C)=0,01$, $C A I C=777,85$ e $E C V I=0,87) ;$ amostra 4 $\left(X^{2} / g l=0,82, G F I=0,94, A G F I=0,91, C F I=\right.$ 0,99 , RMSEA $(90 \% / C)=0,00$, CAIC $=$ $712,12$ e $E C V I=1,59)$.

TABELA 1.

Comparação da estrutura fatorial da escala de sexismo ambivalente em diferentes amostras.

\begin{tabular}{|c|c|c|c|c|c|c|c|c|c|c|}
\hline MODELOS & $\chi^{2}$ & gl & $\chi^{2 / g l}$ & GFI & AGFI & CFI & RMR & RMSEA & CAIC & ECVI \\
\hline \multicolumn{11}{|l|}{ Amostra 1} \\
\hline Unifatorial & 382,49 & 173 & 2,21 & 0,86 & 0,80 & 0,80 & 0,08 & $\begin{array}{c}0,07 \\
(0,06-0,07)\end{array}$ & 915,25 & $\begin{array}{c}1,90 \\
(1,71-2,11)\end{array}$ \\
\hline Bifatorial & 172,83 & 171 & 1,01 & 0,95 & 0,92 & 0,99 & 0,05 & $\begin{array}{c}0,01 \\
(0,00-0,03)\end{array}$ & 718,91 & $\begin{array}{c}1,18 \\
(1,17-1,30)\end{array}$ \\
\hline \multicolumn{11}{|l|}{ Amostra 2} \\
\hline Unifatorial & 239,24 & 167 & 1,43 & 0,89 & 0,84 & 0,94 & 0,11 & $\begin{array}{c}0,05 \\
(0,03-0,06)\end{array}$ & 985,24 & $\begin{array}{c}2,04 \\
(1,85-2,26)\end{array}$ \\
\hline Bifatorial & 144,26 & 163 & 0,89 & 0,94 & 0,91 & 1,00 & 0,08 & $\begin{array}{c}0,01 \\
(0,00-0,02)\end{array}$ & 712,45 & $\begin{array}{c}1,60 \\
(1,57-1,76)\end{array}$ \\
\hline \multicolumn{11}{|l|}{ Amostra 3} \\
\hline Unifatorial & 252,62 & 165 & 1,53 & 0,94 & 0,90 & 0,94 & 0,08 & $\begin{array}{c}0,05 \\
(0,03-0,07)\end{array}$ & 863,82 & $\begin{array}{c}1,13 \\
(1,02-1,16)\end{array}$ \\
\hline Bifatorial & 159,70 & 164 & 0,97 & 0,96 & 0,94 & 1,00 & 0,01 & $\begin{array}{c}0,04 \\
(0,00-0,06)\end{array}$ & 777,85 & $\begin{array}{l}0,87 \\
(0,80-0,97)\end{array}$ \\
\hline \multicolumn{11}{|l|}{ Amostra 4} \\
\hline Unifatorial & 254,96 & 163 & 1,56 & 0,88 & 0,82 & 0,86 & 0,09 & $\begin{array}{c}0,05 \\
(0,04-0,07)\end{array}$ & 821,81 & $\begin{array}{c}2,19 \\
(1,99-2,30)\end{array}$ \\
\hline Bifatorial & 132,67 & 161 & 0,82 & 0,94 & 0,91 & 0,99 & 0,01 & $\begin{array}{c}0,00 \\
(0,00-0,01)\end{array}$ & 712,12 & $\begin{array}{c}1,59 \\
(1,53-1,79)\end{array}$ \\
\hline
\end{tabular}

Notas: $p>0,05$ 
Todas as saturações (Lambdas, $\lambda$ ) estão dentro do intervalo esperado $|0-1|$, denotando não haver problemas de estimação proposta. Além disso, todas são estatisticamente diferentes de zero $(t>1,96$, $p<0,05)$ corroborando a existência de dois fatores para se avaliar 0 sexismo ambivalente: o sexismo hostil - expressão flagrante do preconceito em relação às mulheres e reflete antipatia e intolerância em relação ao seu papel como figura de poder e decisão (por exemplo, As mulheres feministas estão fazendo exigências completamente sem sentido aos homens; $A$ maioria das mulheres não aprecia completamente tudo o que os homens fazem por elas); e o sexismo benévolo refere-se a uma atitude positiva, aparentemente não preconceituosa em relação à mulher, porém descrevendo-a como pessoa frágil, necessitando de atenção e etc. (por exemplo, As mulheres devem ser queridas e protegidas pelos homens; Muitas mulheres se caracterizam por uma pureza que poucos homens possuem); em todas as amostras, observaram-se lambdas positivos entre as duas dimensões - Sexismo hostil e benévolo - (N1 $\lambda=0,30$; N2 $\lambda$ 0,37; N3 $\lambda=$ $0,58$; N4 $\lambda=0,28)$, bem como, os alfas de Cronbach variaram para amostra de 0,79 a 81 para o sexismo hostil e de 0,74 a 0,85 para o sexismo benévolo.

A título de lembrança, a bidimensionalidade apresentada pelo sexismo ambivalente, expressa um conjunto de estereótipos quanto à avaliação cognitiva, afetiva e atitudinal sobre o papel apropriado que cada indivíduo deve ocupar ou executar na sociedade (Glick \& Fiske, 1996), desta forma, a relação entre as dimensões do sexismo (apresentando lambdas $(\lambda)$ positivos entre as dimensões), refere-se que na existência de que uma das formas de sexismo, possivelmente, poderá influenciar a outra forma discriminatória frente às mulheres.
Esses resultados confirmam, empiricamente, os resultados já encontrados por Ferreira (2004), Formiga, Gouveia e Santos (2002) e Formiga (2005) em sua análise exploratória, bem como, no estudo transcultural de Glick e cols. (2000); mas, também, revelou, considerando 0 estudo de confirmatório de Formiga (2010), que 0 inventário, ainda mensura 0 preconceito frente às mulheres em termos temporais e geopolíticos, destacando que é fiel a organização item-fator de acordo como - que se esperava para o Sexismo Benévolo (por exemplo, ISA 01, ISA 03, ISA 06, ISA 08, ISA 09, ISA 12, ISA 13, ISA 17, ISA 19, ISA 20, ISA 22) e para o Sexismo Hostil (por exemplo, ISA 02, ISA 04, ISA 05, ISA 07, ISA 10, ISA 11, ISA 14, ISA 15, ISA 16, ISA 18, ISA 21).

\section{DISCUSSSAO}

Além de comprovar a existência do sexismo ambivalente em sua bi-dimensionalidade, os indicadores psicométricos encontrados neste estudo aponta para uma melhor estrutura do inventário do que a encontrada por Formiga, Gouveia e Santos (2002); atenta-se, tanto para a acurácia da mensuração do sexismo ambivalente quanto a reflexão teórica de que esse fenômeno existe e vem se mantendo, direta - em sua forma hostil - ou indiretamente em sua forma sutil frente as mulheres brasileiras.

De forma geral, a forma do sexismo hostil deve ser inibida, pois é possível que a partir deste decorra-se a violência em relação às mulheres; e quanto ao surgimento do sexismo benévolo? Este merece, também, se não inibi-lo, porque é sutil e tem sido um problema grande quanto a identificação dele nas relações interpessoais e que ao apontá-lo e deflagrar a extinção do mesmo, este, parece escorrer por entre os dedos, pois confunde-se com processos e praticas cavalheiresca, sensível 
e romantizada das relações entre homens e mulheres. O fato é que, com essas novas formas preconceituosas, a discriminação contra a mulher vem sendo camuflada e carregada de finas e dissimuladas atitudes. 0 que atualmente encontra-se, ao acompanhar o cotidiano brasileiro, o qual parece confirmar praticamente o óbvio, é que 0 elogio ou gratidão diante da mulher muitas vezes passa a ser a outra cara da moeda há muito conhecida: o sexismo sutil (Swim, Mallett \& Stangor, 2004; Thomas \& Esses, 2004).

Mesmo com todas as mudanças na sociedade civil brasileira, embasadas na Constituição de 1988, principalmente no que se refere ao preconceito - especificamente, quanto à igualdade entre homens $e$ mulheres - tomado como princípio geral, ainda é possível acompanhar um avanço em relação às oportunidades e os direitos das mulheres, mas não a superação da desigualdade (Marodin, 1997), condição corroborada neste estudo.

A comprovação da organização dos itens em seus respectivos fatores e a sua consistência interna do inventario aqui avaliado não permitirá acabar com as atitudes discriminatórias, porém, espera-se oferecer base para conhecer a amplitude desse problema, bem como, predizer conseqüências futuras. Em resumo, considerando os indicadores de bondade de ajuste, houve evidência da validade fatorial e da consistência interna para o inventário de sexismo ambivalente em todas as amostras, justificando seu emprego no contexto brasileiro.

Todavia, um limite neste estudo merece ser destacado: seria de extrema importância conhecer os aspectos que podem ser comuns a todas as culturas e aqueles que são específicos, contribuindo para consolidar um marco teórico do sexismo ambivalente; não menos útil, reunir evidências adicionais de sua validade e precisão intra, inter e pan-culturais, por exemplo, validade de critério ou convergente em relação a construtos correlatos, bem como, conhecer sua estabilidade temporal (teste-reteste), comparando com os resultados que podem ser indicados por outros autores; a replicabilidade do inventário, considerando amostras maiores e mais diversificadas quanto às características dos participantes, incluindo também jovens de diferentes contextos socioculturais e econômicos, bem como, seus familiares, seria importante para avaliar 0 processo socializador do preconceito frente às mulheres.

\section{REFERENCIAS}

Aguiar, N. (1997). Gênero e ciências humanas: desafio às ciências desde a perspectiva das mulheres. Rio de Janeiro: Rosa dos tempos.

Byrne, B. M. (1989). A primer of LISREL: Basic applications and programming for confirmatory factor analytic models. New York: Springer-Verlag

Ferreira, M. C. (2004). Sexismo hostil e benevolente: inter-relações e diferenças de gênero. Temas em Psicologia, 12 (21), 119-126.

Formiga, N. S. \& Camino, L. (2001). A Dimensão do Inventário de Papeis Sexuais (BSRI): A masculinidade e feminilidade em universitários. Estudos de Psicologia,18, (2), 41-49.

Formiga, N. S. (2005). Estrutura fatorial do inventário de sexismo ambivalente a partir da análise dos eixos principais. Psicólogo Informação, 9 (9), 09-28.

Formiga, N. S., Gouveia, V. V. \& Santos, M. N. (2002). Inventário de sexismo ambivalente: sua adaptação e relação com o gênero. Revista Psicologia em estudo, 7 (1),105-111.

Formiga, N. S.; Yepes, C. F.; Alves, I.; Ayroza, I.; Teixeira, J. \& Curado, F. (2004). Flagrando o preconceito: Uma análise descritiva das atitudes preconceituosas frente aos negros, mulheres e homossexuais. Anais do XXXIV reunião anual da sociedade 
brasileira de psicologia. Formação do psicólogo brasileiro: História de desafios e conquistas. Ribeirão Preto SP. 26 a 29 de Outubro. [Resumo Eletrônico].

Formiga, N. S. (2011). Inventário de sexismo ambivalente: Um estudo a partir da modelagem de equação estrutural. Mimeo.

Garson, G. D. (2003). PA 765 Statnotes: An online textbook. Endereço de página Web:

http://www2.chass.ncsu.edu/garson/pa 765/statnote.htm (consultado dia 17 de maio de 2005).

Glick, P. \& Fiske, S. T. (1996). The Ambivalent Sexism Inventory: Differentiating hostile and benevolent sexism. Journal of Personality and Social Psychology, 70(3), 491-512.

Glick, P. \& Fiske, S. T. (2001). Ambivalent stereotypes as legitimizing ideologies: Differentiating paternalistic and envious prejudice. In J. T. Jost \& B. Major (Eds.) The psychology of legitimacy: Ideology, justice, and intergroup relations (pp. 278-306). New

York: Cambridge University Press

Glick, P., Fiske, S. T., Mladinic, A., Saiz, J. L., Abrams D., Masser, B. e cols. (2000). Beyond prejudice as simple antipathy: Hostile and benevolent sexism across cultures. Journal of Personality and Social Psychology, 79 (5), 763-775.

Hair, J. F.; Tatham, R. L.; Anderson, R. E.; Black, W. (2005). Análise Multivariada de Dados. Porto Alegre: Bookman.

Joreskög, K., \& Sörbom, D. (1989). LISREL 7 user's reference guide. Mooresville: Scientific Software.

Kelloway, E. K. (1998). Using LISREL for structural equation modeling: $A$ researcher's guide. Thousand Oaks, CA: Sage Publications.

Marodin, M. (1997). As relações entre o homem e a mulher na atualidade. Em: Marlene N. Strey (Org.). Mulher:
Estudos de gênero (pp.09-18). São Leopoldo: Unisinos.

Munanga, K. (2002). Prefácio. Em: I. Carone e M. A. S. Bento (Orgs.). Psicologia social do racismo: Estudos sobre branquitude e branqueamento no Brasil (pp. 9-11). Petrópolis: Vozes.

Pettigrew, T. F. \& Meertens, R. W. (1995). Subtle and blatant prejudice in Western Europe. European journal of social psychology, 25, 57-75.

Siano, J. A. (2000). Constituição da Republica Federativa do Brasil. São Paulo: Editora Rideel.

Swim, J. K., Mallett, R. \& Stangor, C. (2004). Understanding Subtle Sexism: Detection and Use of Sexist Language. Sex Roles, 51 (3/4), 117-128.

Swin, J. K., Aikin, K. J., Hall, W. S. \& Hunter, B. A. (1995). Sexism and racism: Oldfashioned and modern prejudices. Journal of Personality and Social Psychology, 68, 199-214.

Tabachnick, B. G. \& Fidel, L. S. (1996). Using multivariate statistics. Harper Collins: New York, NY.

Thomas, C. A. \& Esses, V. M. (2004). Individual differences in reactions to sexist humor. Group Processes \& Intergroup Relations, 7 (1), 89-100.

Tougas, F., Brown, R., Beaton, A. N. \& Joly, S. (1995). Neosexism: Plus ça change, Plus c'est pareil. Personality and social psychology behavior, 21 (8), 842-849.

Van de Vijver, F.; Leung, K. (1997). Methods and data analysis for cross-cultural research. Thousand Oaks, CA: Sage Publications. 
ANEXO:

\section{INVENTARIO DE SEXISMO AMBIVALENTE (ISA)}

ISA 01. Ainda que um homem tenha muito êxito em sua vida, não poderá sentir-se completo a menos que tenha o amor de uma mulher.

ISA 02. Com o pretexto da igualdade, muitas mulheres buscam privilégios especiais, como condições de trabalho que as favoreçam.

ISA 03. Em caso de grandes ou pequenos acidentes, as mulheres devem ser resgatadas antes que os homens.

ISA 04. A maioria das mulheres interpreta os comentários ou brincadeiras inocentes como sexistas, isto é, como expressões preconceituosas ou discriminatórias contra elas.

ISA 05. As mulheres se ofendem muito facilmente.

ISA 06. As pessoas não podem ser verdadeiramente felizes em suas vidas a menos que tenham uma outra pessoa do sexo oposto. (Ex.: para o homem, uma mulher, e vice-versa).

ISA 07. Na verdade, o que as mulheres feministas pretendem é que a mulher tenha mais poder que 0 homem.

ISA 08. Muitas mulheres se caracterizam por uma pureza que poucos homens possuem.

ISA 09. As mulheres devem ser queridas e protegidas pelos homens.

ISA 10. A maioria das mulheres não dá valor completamente a tudo o que os homens fazem por ela.

ISA 11. As mulheres tentam ganhar poder controlando os homens.

ISA 12. Todo homem deve ter uma mulher a quem amar.

ISA 13. O homem está incompleto sem a mulher.

ISA 14. As mulheres exageram os problemas que têm no trabalho.

ISA 15.Uma vez que uma mulher consiga que o homem se comprometa com ela, geralmente, ela tenta controlar.

ISA 16. Quando as mulheres são vencidas pelos homens numa disputa justa, geralmente se queixam de serem "roubadas" ou discriminadas.

ISA 17. Uma boa mulher deveria ser posta em um pedestal pelo homem.

ISA 18. Existem muitas mulheres que, para chamar a atenção de um homem, primeiro se insinuam sensualmente e depois rejeitam seus avanços ou 'cantadas'.

ISA 19. As mulheres, em comparação com os homens, tendem a ter uma maior sensibilidade moral.

ISA 20. Os homens deveriam estar dispostos a sacrificar seu próprio bem-estar a fim de dar segurança econômica e social às mulheres.

ISA 21. As mulheres feministas estão fazendo exigências completamente irracionais aos homens.

ISA 22. As mulheres, em comparação com os homens, mostram um sentido mais refinado para a cultura e o bom gosto. 